\title{
Preparation of erosion and deposition investigations on plasma facing components in Wendelstein 7-X
}

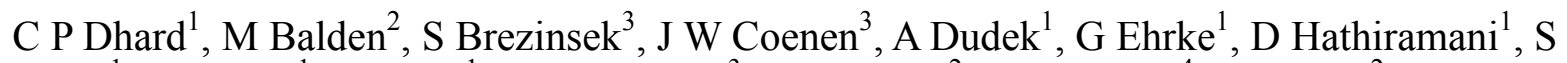

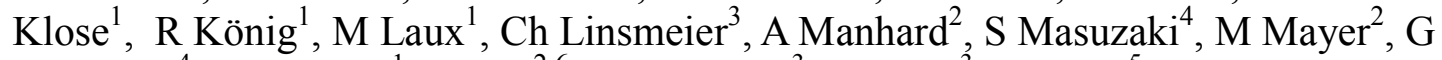

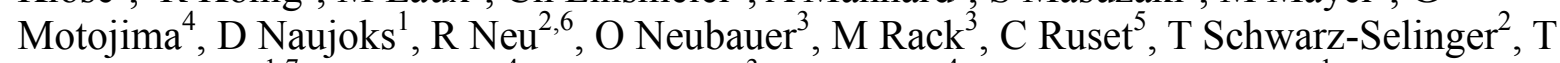 \\ Sunn Pedersen ${ }^{1,7}$, M Tokitani $^{4}$, B Unterberg ${ }^{3}$, M Yajima ${ }^{4}$ and The W7-X Team ${ }^{1}$ \\ ${ }^{1}$ Max-Planck-Institut fuer Plasmaphysik, Wendelsteinstrasse 1, 17491 Greifswald, Germany
${ }^{2}$ Max-Plank-Institut fuer Plasmaphysik, Boltzmannstrasse 2, 85748 Garching, Germany
${ }^{3}$ Forschungszentrum Juelich GmbH, Institut fuer Energie- und Klimaforschung - Plasmaphysik, Part
Trilateral Euregio Cluster (TEC), 52425 Juelich, Germany
${ }^{4}$ National Institute for Fusion Science, 322-6 Oroshi, Toki 509-5292, Japan
${ }^{5}$ National Institute for Laser, Plasma and Radiation Physics, 077125 Magurele-Bucharest, Romania
${ }^{6}$ Technische Universität München, Boltzmannstr. 15, 85748 Garching, Germany
${ }^{7}$ Ernst-Moritz-Arndt-Universität Greifswald, Domstraße 11, 17489 Greifswald, Germany
}

Email: dhard@ipp.mpg.de

In the Wendelstein 7-X stellarator with its twisted magnetic geometry the investigation of plasma wall interaction processes in 3D plasma configurations is an important research subject. For the upcoming Operation Phase i.e. OP1.2, three different types of material probes have been installed within the plasma vessel for the erosion/deposition investigations in selected areas with largely different expected heat load levels, namely, $\leq 10 \mathrm{MW} / \mathrm{m}^{2}$ at the Test Divertor Units (TDU), $\leq 500$ $\mathrm{kW} / \mathrm{m}^{2}$ at the baffles, heat shields and toroidal closures and $\leq 100 \mathrm{~kW} / \mathrm{m}^{2}$ at the stainless steel wall panels. These include 18 exchangeable target elements at TDU, about 30000 screw heads at graphite tiles and 44 wafer probes on wall panels, coated with marker layers. The layer thicknesses, surface morphologies and the impurity contents were pre-characterized by different techniques and subjected to various qualification tests. The positions of these probes were fixed based on the strike line locations on the divertor predicted by field line diffusion and EMC3/EIRENE modeling calculations for the OP1.2 plasma configurations and availability of locations on panels in direct view of the plasma. After the first half of the operation phase i.e. OP1.2a the probes will be removed to determine the erosion/deposition pattern by post-mortem analysis and replaced by a new set for the second half of the operation phase, OP1.2b.

Keywords: Wendelstein 7-X, Plasma facing components, Plasma wall interaction, Erosion and deposition

\section{Introduction}

Wendelstein 7-X (W7-X) is an optimized 3D helical shaped stellarator with 5 fold symmetry (see figure 1). It is designed for steady state plasma operation of up to 30 min with $10 \mathrm{MW}$ Electron Cyclotron Resonance Heating (ECRH) power. For physics experiments of up to $10 \mathrm{~s}$ duration ion cyclotron resonance (4 MW) and neutral beam injection (20 MW) heating systems are being stepwise installed starting with OP1.2 $[1,2]$. In future steady state operated fusion power plants, Plasma Wall Interaction (PWI) is expected to play a substantial role. Important processes are, e.g. erosion and re-depostion of wall materials, plasma impurity contamination and impurity exhaust from plasma etc. Extensive PWI studies have been performed on various fusion devices $[3,4]$. In the tokamaks, the wall geometry is toroidally symmetrical around the torus, however in stellarators, in particular in W7-X, with its island divertor and 3D twisted plasma configurations, PWI and its resulting impurity migration can be expected being much more challenging to describe and understand.

As for Plasma Facing Components (PFC), W7-X is equipped with 10 discrete island divertors with baffles, toroidal \& poloidal divertor closures, carbon tiled wall protection heat shields and Stainless Steel (SS) wall and divertor pumping gap panels (see figure 2). In the upcoming 
Operation Phase (OP1.2), starting Sept. 2017, the divertor consists of 10 inertially cooled, fine grain graphite Test Divertor Units (TDU), which are identical in shape to the actively water cooled CFC high heat flux divertor, being used in the next operation phase OP2. Between OP1.2a and OP1.2b, in addition two so called scraper elements (essentially target modules) will be installed just in front of one upper and one lower TDU divertor module, to investigate their ability to mitigate possible thermal overloads of target tiles at the pumping gap edge in certain magnetic configurations when the bootstrap current evolution may drive strike lines from the horizontal onto the vertical target area [5]. To reach sufficient pumping capability for quasi-continuous plasma operation in OP2, ten cryo-vacuum pumps will be installed behind the divertors.

During plasma operation the PFCs are subjected to heat loads varying from $\leq 10 \mathrm{MW} / \mathrm{m}^{2}$ at the divertor target, $\leq 500 \mathrm{~kW} / \mathrm{m}^{2}$ at C-tiles (baffle, heat shield and toroidal closure) and $\leq 100 \mathrm{~kW} / \mathrm{m}^{2}$ at the outer wall panels. This provides an opportunity to carry out material erosion and deposition measurements at these different load levels and to perform material migration estimations within the 3D geometry of W7-X, in OP1.2 not yet requiring complicated, actively cooled material probes. Marker layers have been coated on probes of different kind and installed at various locations. The PWI modeling activities are being taken up in parallel, correlating the codes dealing with the plasma configuration i.e. EMC-EIRENE with the wall interaction i.e. ERO2.0 for the 3D geometry. These models then will be used to interpret the measured experimental data. The break between OP1.2a and OP1.2b will be used to exchange all probes and a set of selected screws by new ones, allowing separate erosion/deposition behavior studies in the two campaigns sections. In the next chapter the details of the selected probe types, as well as their design and qualification tests, etc. are being discussed.

\section{PWI target elements at the TDU}

Each TDU is made of 9 horizontal and 3 vertical Target Modules (TM) and each TM consists of a certain number of Target Elements (TE) as shown in figure 3a. Based on the strike line positions for the plasma configurations to be used in OP1.2, 18 TEs were chosen for PWI studies. The main drive behind choosing the locations was to map the erosion/deposition pattern at an identical location on all 10 divertor modules (TM2h in fig. $3 \mathrm{~b}$ ) as well as on one upper and one lower divertor module within W7-X module 5 (all marked TEs in fig. 3b).

These 18 TEs were specially designed for easy exchange. The 18 TEs were put together from altogether 132 graphite pieces of 34 different sizes. The graphite pieces were mounted onto Ti-ZrMo (TZM) supports, forming special exchangeable TEs, which can be easily installed at the 18 locations. In order to coat these graphite pieces with C/Mo marker layers, a special Combined Magnetron Sputtering and Ion Implantation (CMSII) [6] coating technique was developed at the National Institute for Laser, Plasma and Radiation Physics, Romania. The main difficulty was associated with the deposition of compact and uniform $\mathrm{C}$ coatings of few $\mu \mathrm{m}$ thickness on a large area. This was achieved by upgrading the magnetron power supply to $1100 \mathrm{~V}$ and optimizing the process parameters to avoid soot formation. The marker layer coating, consisting of an about $7 \mu \mathrm{m}$ thick C layer with a 0.2-0.4 $\mu \mathrm{m}$ interlayer of Mo, was deposited on an area with $64 \times 23 \mathrm{~mm}^{2}$ and positioned using special masks, in the middle of the graphite pieces (see figure 4). The layer thicknesses, surface morphology and impurity contents were measured by Scanning Electron Microscope (SEM), Energy Dispersive X-ray spectroscopy (EDX) and Rutherford Backscattering Spectrometry (RBS) for pre-characterization and high heat flux tests were performed for the qualification. SEM images of the sample prepared with the fracture through the C/Mo marker layer showed the internal structure of the $\mathrm{C}$ coating to be compact, featureless, very dense and without pores or cracks. EDX analyses performed at $25 \mathrm{keV}$, indicated an impurity content of $\mathrm{O}<2$ at. \%, $\mathrm{W}<0.1$ at. $\%$ and $\mathrm{Ar}<0.1$ at. \%. RBS measurements using $2.5 \mathrm{MeV}$ protons at a scattering angle of $165^{\circ}$ in the BOMBARDINO/BesTec facility at Max-Planck Institute for Plasma Physics (IPP) Garching confirmed the coating thickness and the impurity contents. The high heat flux tests were performed at the GLADIS test facility at IPP Garching using a $26 \mathrm{keV} \mathrm{H}$ ion-beam for applying 50 pulses of $8 \mathrm{MW} / \mathrm{m}^{2}$ heat load with pulse durations of $2.5 \mathrm{~s}$ without any delamination or visible damage, only few micro cracks were observed by SEM. All 132 graphite pieces were cleaned at the manufacturer's workshop by baking at $1200^{\circ} \mathrm{C}$ for $24 \mathrm{~h}$ in a vacuum furnace at $1 \times 10^{-4}$ mbar. Furthermore, before installation in the TDU, all assembled TEs were in addition again baked at 
$550^{\circ} \mathrm{C}$ for $8 \mathrm{~h}$ in a vacuum furnace at $1 \times 10^{-4} \mathrm{mbar}$ at IPP Greifswald. All exchangeable TEs have been installed in the TDUs as planned (figure 4).

After OP1.2a, all these TEs will be removed to determine the erosion/deposition pattern by post-mortem analysis and replaced for OP1.2b by new ones, some of which also comprising of thin $\mathrm{W}$ in addition to the $\mathrm{C}$ coatings.

\section{TZM Screws}

Heat shield graphite tiles cover almost the entire inboard toroidal side of the W7-X plasma vessel and the whole poloidal plasma circumference at certain toroidal locations. Also the divertor baffle structures at the inboard and outboard side, which are supposed to improve the containment of the neutral particles in the divertor, as well as the toroidsal divertor closure at one divertor end, are made of such tiles. TZM (Ti 0.5\%, Zr 0.08\%, Mo 99.39\%) screws, as shown in figures 4 and 5, are used to fix the graphite tiles to water cooled (for OP2) $\mathrm{CuCrZr}$ heat sink plates. The numbers of screws used for fitting all tiles are shown in table 1. With each screw head facing the plasma having a surface area of about $1 \mathrm{~cm}^{2}$, the overall surface area of all screws adds up to about $3 \mathrm{~m}^{2}$. In order to avoid Mo contamination of the plasma, all screws heads were coated with a well characterized amorphous carbon layer, providing the additional unique opportunity of using these screw heads also as PWI probes for large scale erosion/deposition pattern mapping.

Table 1. Number of TZM screws of different lengths used for heat shield, baffle and toroidal closure tiles.

\begin{tabular}{llll}
\hline & M6x16 & M6x20 & M6x18 \\
\hline Heat shield & 5871 & 8275 & 12 \\
Baffle, Toroidal closure & 20 & 15406 & 40 \\
Total (29624) & 5891 & 23681 & 52 \\
\hline
\end{tabular}

Various coating materials were tried and subjected to the qualification tests until finally an amorphous Carbon (a-C) coating was chosen with a coating thickness in the range of 6-15 $\mu \mathrm{m}$ having a $1 \mu \mathrm{m}$ Ti interlayer. The coating of the screws and some of the qualifications tests were carried out at Fraunhofer IST Braunschweig. A SEM image of the a-C coating is shown in figure 6, showing a dense and defect free coating. EDX and SIMS showed a small Argon contamination, caused by using Ar as working gas during the coating process. The adhesion test of the coating showed no delamination up to $20 \mathrm{~N}$, which was acceptable. Heat load tests at $500 \mathrm{~kW} / \mathrm{m}^{2}$ over 100 $\mathrm{s}$ showed the graphite surface to remain intact for the observed surface temperature of $930^{\circ} \mathrm{C}$ [7]. The coating thickness was measured using a Betascope (Helmut Fischer $\mathrm{GmbH}$ ) making use of a ${ }^{147} \mathrm{Pm}$ beta source and back-scattering measurement with a Geiger-Müller counter. The layer thickness is determined from the difference in back-scattering from the $\mathrm{C}$ coating and the TZM substrate materials. The measurements were made by Betascope probe fitted to a Fischerscope ${ }^{\circledR}$. For coating, 4 large carrier plates with each about 1100 screws were simultaneously processed. The large plate sizes caused a continuous variation of the layer thickness over the carrier plates, which could be well characterized by measurements at selective distributed locations combined with fitting, allowing the determination of the thickness profile distribution across all screws on a board. Screws from the individual carrier plates were then sorted into separate boxes within thickness steps of $0.5 \mu \mathrm{m}$. Each box of one thickness step served one heat shield, thereby making the documentation rather easy.

All the screws have been mounted with a special tool with a predefined torque. In order to estimate the erosion/deposition pattern, the coating thickness on selected screw heads will be measured in-situ after OP1.2a and OP1.2b once the plasma vessel will be accessible to enter. The Betascope front part will be modified such that the screw heads within various tile geometries are accessible for the measurement. Furthermore, about 500 screws will be completely exchanged, for full post mortem analysis in the lab. 


\section{Wafer probes}

For installation on SS wall panels and the pumping gap panels of the TDU, $44 \mathrm{Si}$ wafer probes have been prepared. Similar kinds of probes have been used in the Joint European Torus (JET) [8] and Axially Symmetric Divertor Experiment (ASDEX) Upgrade [9] tokamaks. The probe locations are under direct plasma view and mounted in W7-X module 4 (see figure 7). The probe heads of the dimensions $11.5 \times 11.5 \times 8 \mathrm{~mm}^{3}$ were screwed onto bolts welded to the SS wall panels. A commercially available Si wafer was cut into pieces to fit into the top slot of probe head (see figure $8 \mathrm{~b}$ ). On some of the wafers, thin layers of $\mathrm{Ni}$ and a-C:H have been coated to proxy the panel material. The details of surface area and coatings are presented in table 2 .

Table 2. The total surface area of wafer probes exposed to plasma and together with the thickness and mass of the coatings, for the bare Silicon wafer (row 1) there is no coating

\begin{tabular}{llll}
\hline Wafer type & Surface area & Coating thickness & Mass of coatings \\
\hline Silicon & $33 \mathrm{~cm}^{2}$ & none & none \\
Ni on $\mathrm{Si}$ & $7.5 \mathrm{~cm}^{2}$ & $\leq 50 \mathrm{~nm}$ & $\mathrm{Ni}: \leq 333.75 \mu \mathrm{g}$ \\
a-C:H on $\mathrm{Si}$ & $9 \mathrm{~cm}^{2}$ & $\leq 100 \mathrm{~nm}$ & $\mathrm{a}-\mathrm{C}: \mathrm{H} \leq 180 \mu \mathrm{g}$ \\
\hline
\end{tabular}

All these probes have already been installed in W7-X (see figure 8a) and at the end of OP1.2a, will be removed and replaced by a different set of probes. In addition to 30 similar Si wafer probes, then also 12 Directional Material Probes (DMP) and 2 cavity probes will be installed for OP1.2b.

The DMPs will be similar to the ones being used in the Large Helical Device (LHD), Japan [10]. The probed will have a circular disc with a pin installed in the middle. From the shadow of the pin formed on the disc, the incident angle of the deposited material will be estimated. Multiple shadows indicate different directions of the incident materials due to perhaps changes in deposition processes or plasma configurations. Unlike the simple $\mathrm{Si}$ wafer probes, these probes will be electrically insulated from the wall panels, this affecting the depositions taking place during plasma operations and glow discharge cleaning. By comparing the deposition patterns during OP1.2a and $1.2 \mathrm{~b}$, such influence is expected to be distinguishable. The 2 cavity probes $[11,12]$ will be installed on the pumping gap panels and will be used to investigate the surface loss probability of hydrocarbon radicals.

\section{Summary and plans with tungsten PFC}

All the probes aimed for the erosion/deposition measurements for OP1.2 have been pre-qualified and pre-tested, cleaned and installed on the respective PFCs within the W7-X plasma vessel. The plasma vessel has been closed and the evacuation started. The replacement of the exchangeable PWI TEs of the TDU and of wafer probes on the wall panels between OP1.2a and OP1.2b are in preparation. The necessary arrangements for the in-situ layer thickness measurements on the TZM screws heads are also being made.

The higher heat load PFCs of W7-X are all made of graphite, however, some very first steps have already been initiated to introduce $\mathrm{W}$ as plasma facing material in W7-X. For OP1.2a, 10 graphite heat shield tiles on the plasma vessel inboard side facing the electron cyclotron resonance heating launchers have been replaced by W coated TZM reflector tiles to improve multi-path absorption of the electron cyclotron waves in the plasma. For OP1.2b, also one exchangeable PWI target element with a short $\mathrm{W}$ coated section in a highly loaded area as well as a few heat shield tiles with $\mathrm{W}$ coating are being prepared.

\section{Acknowledgments}

The coating and testing of TZM screws were carried out by M. Keunecke and his team at Fraunhofer IST Braunschweig. This work has been carried out within the framework of the EUROfusion Consortium and has received funding from the Euratom research and training program 
2014-2018 under grant agreement No 633053. The views and opinions expressed herein do not necessarily reflect those of the European Commission.

\section{References}

[1] Bosch H-S et al, 2015 Experience with the commissioning of the superconducting stellerator Wendelstein 7-X, Fusion Engineering and Design 96-97 22-27

[2] Pedersen T S et al, 2017 Key results from the first plasma operation phase and outlook for future performance in Wendelstein 7-X, Phys. Plasmas 24055503.

[3] Federici $\mathrm{G}$ et al, 2001 Plasma-material interactions in current tokamaks and their implications for next step fusion reactors, Nuclear Fusion 41 1967-2137.

[4] Roth J et al, 2009 Recent analysis of key plasma wall interactions issues for ITER, Journal of Nuclear Materials 390-391 1-9

[5] Hölbe $\mathrm{H}$ et al, 2016 Access to edge scenarios for testing a scraper element in early operation phases of Wendelstein 7-X, Nuclear Fusion 56026015.

[6] Ruset C et al 2009 Industrial scale $10 \mu \mathrm{m} \mathrm{W}$ coating of CFC tiles for ITER-like Wall Project at JET, Fusion Engineering and Design 84 1662-1665

[7] Greuner $\mathrm{H}$ et al 2003 Final design of W7-X divertor plasma facing components-tests and thermosmechanical analysis of baffle prototypes, Fusion Engineering and Design 66-68 447-452

[8] Mayer M et al, 1997 Erosion at the vessel walls of JET, Journal of Nuclear Materials 241-243 469475

[9] Rohde $\mathrm{V}$ et al, 2005 Carbon erosion and a:C-H layer formation at ASDEX Upgrade, Journal of Nuclear Materials 337-339 847-851

[10] Masuzaki S, 2013 Directional Material Probe for Deposition Layer Studies, Plasma and Fusion Research 81202110

[11] Mayer $\mathrm{M}$ et al, 2006 Further insight into the mechanism of hydrocarbon layer formation below the divertor of ASDEX Upgrade, Nuclear Fusion 46 914-920

[12] Krat $S$ et al, 2015 Hydrocarbon film deposition inside cavity samples in remote areas of the JET divertor during the 1999-2001 and 2005-2009 campaigns, Journal of Nuclear Materials 463 822826 


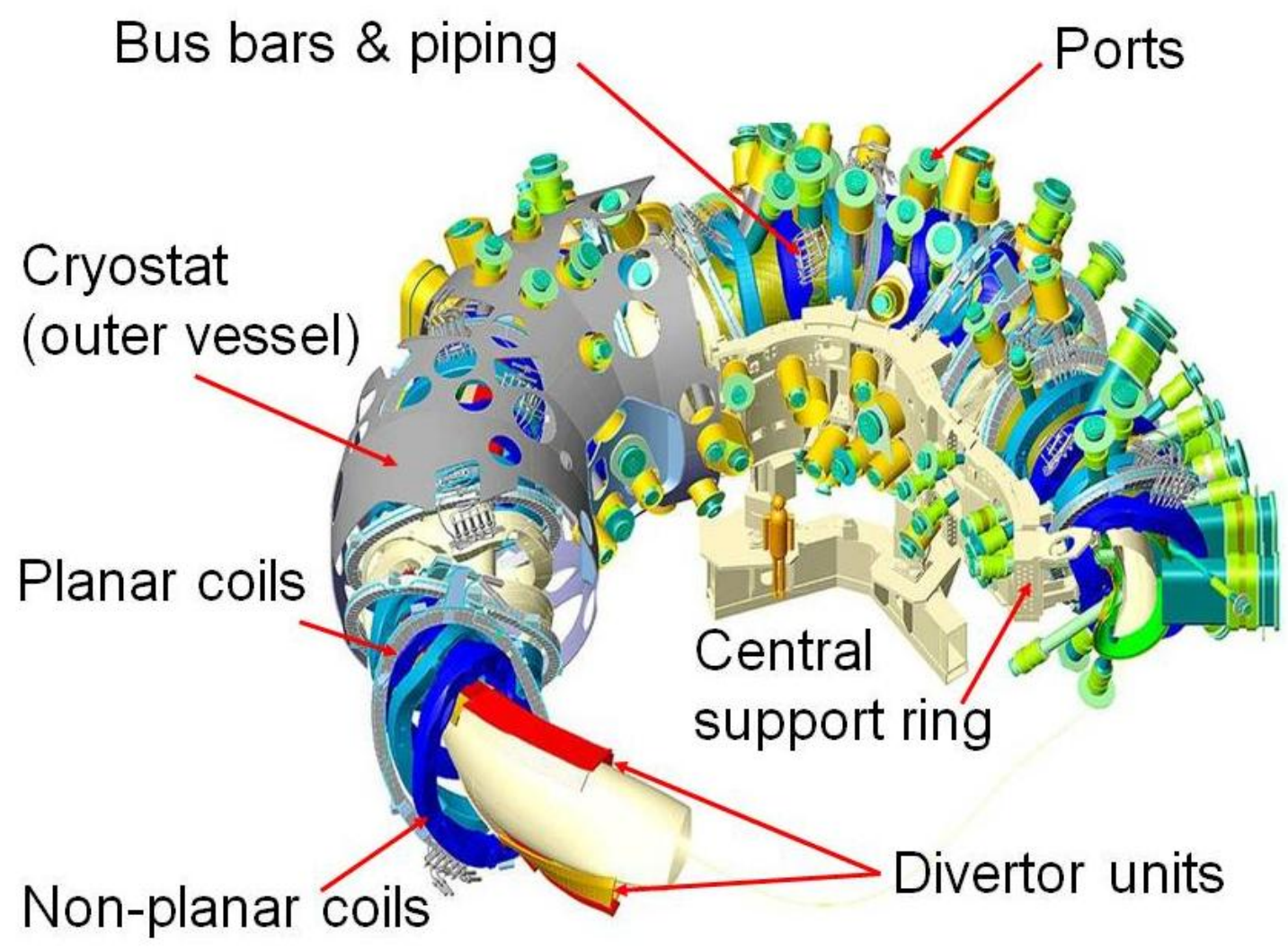

Figure 1. Overview of W7-X stellarator, showing various components, non-planar, planar coils and bus bars are made of $\mathrm{NbTi}$ super conductor

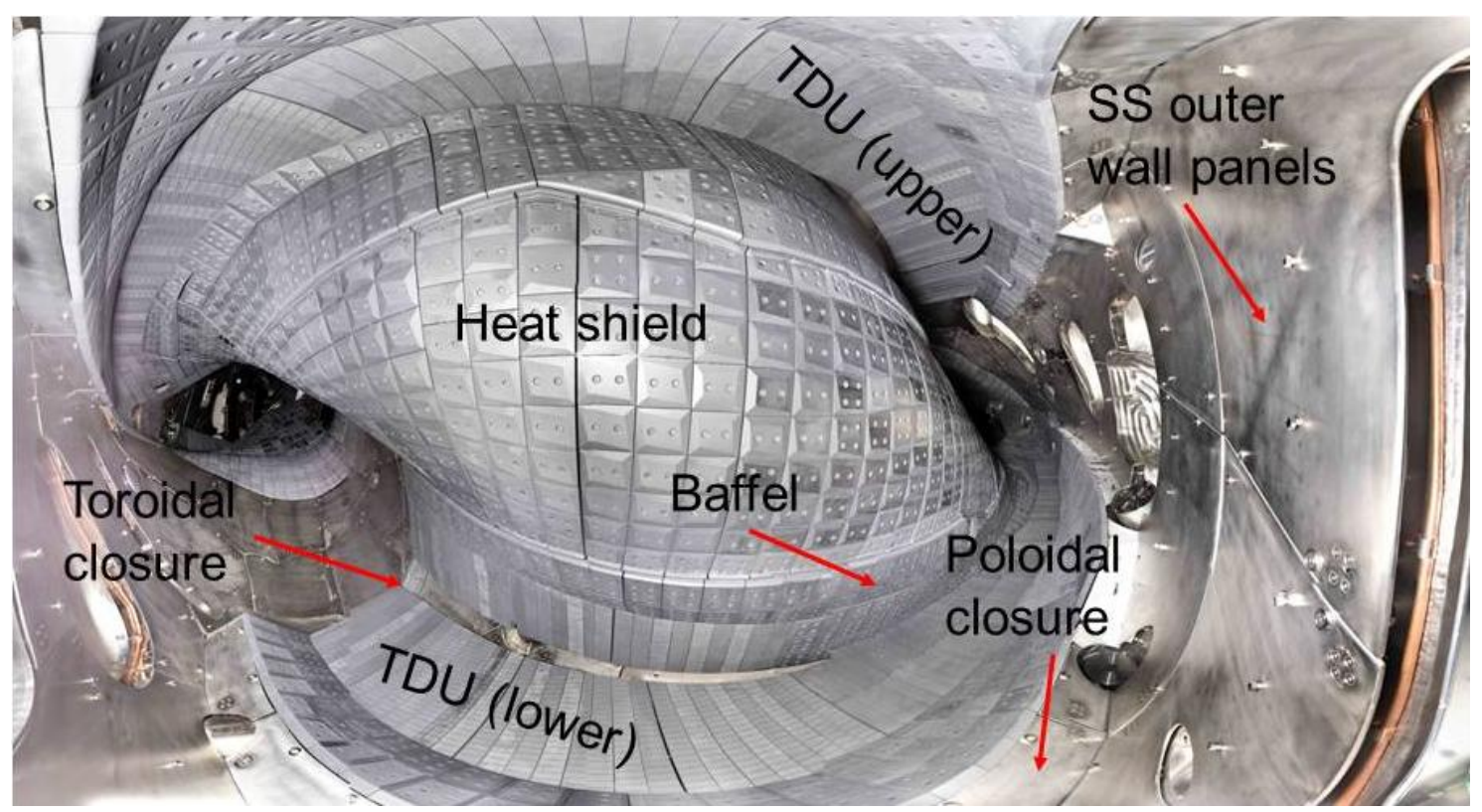

Figure 2. View into W7-X plasma vessel from module 4, showing the installed plasma facing components (photo from T. Braeuer) 


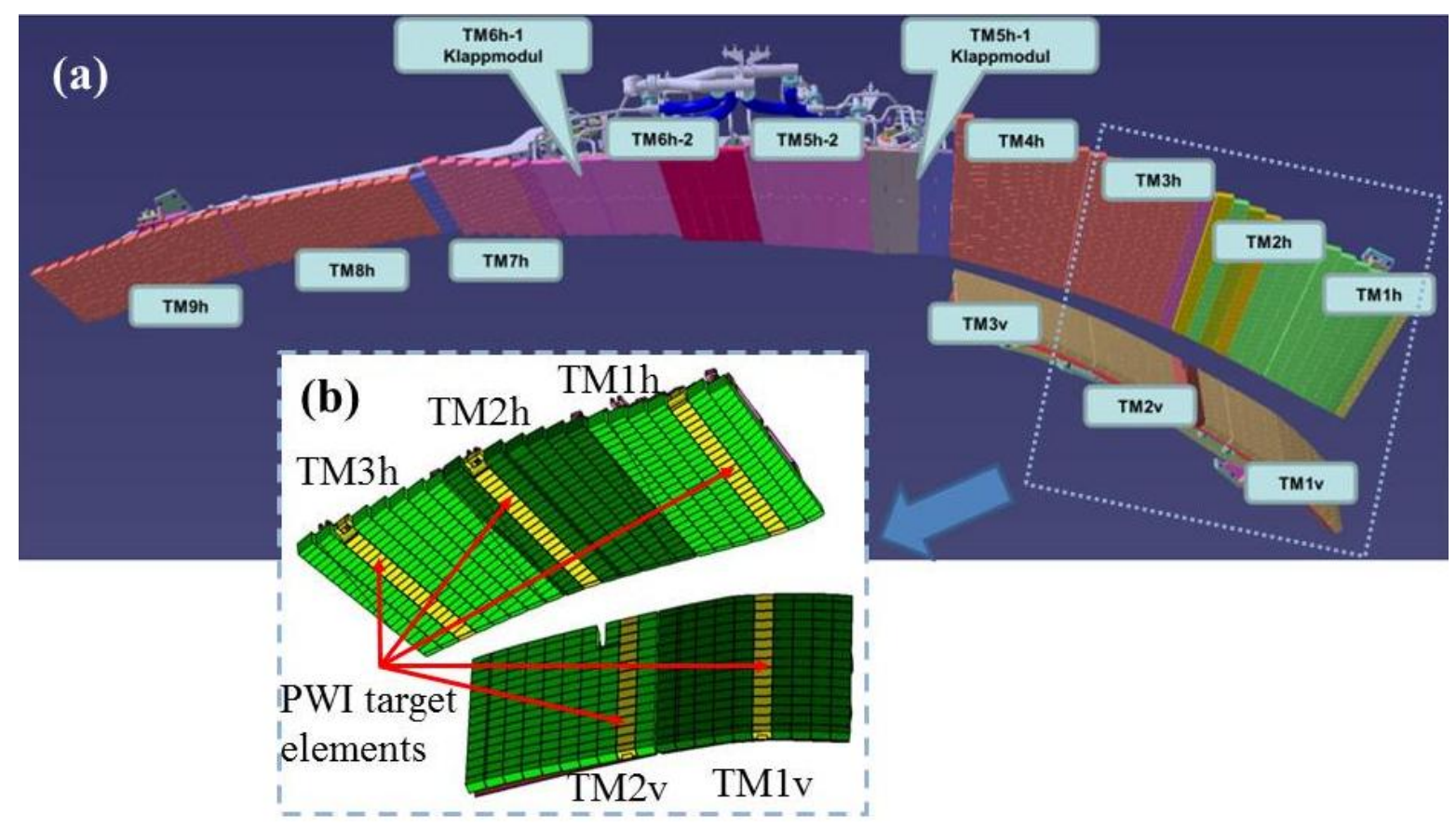

Figure 3. (a) Schematics of TDU showing 9 horizontal (TM1h...TM9h) and 3 vertical (TM1v...TM3v) target modules, (b) enlarged view of 3 horizontal and 2 vertical target modules shown with PWI target elements with marker layer coatings

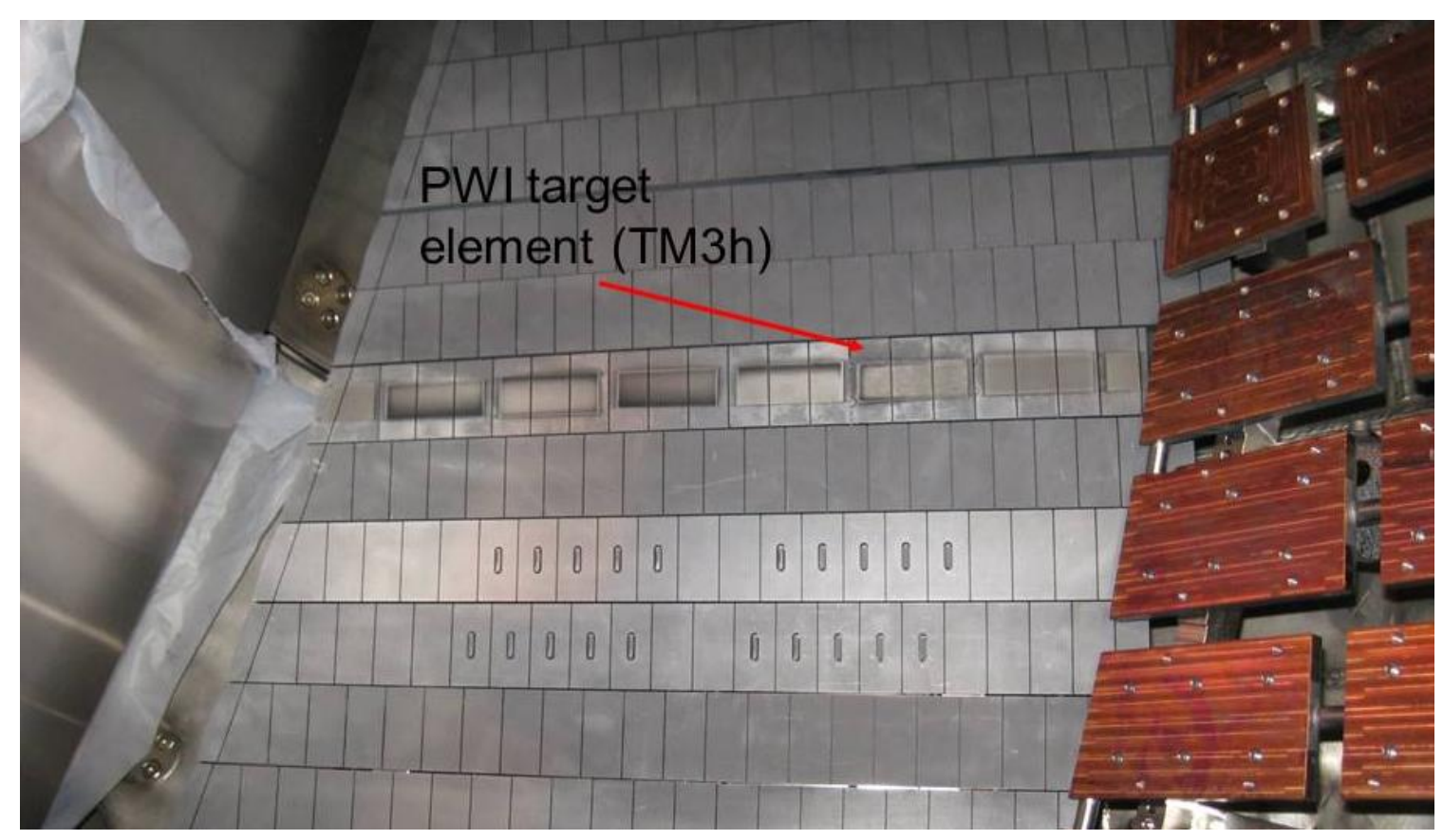

Figure 4.Assembly of exchangeable PWI target element TM3h mounted in TDU, marker layer coating in the rectangle shaped segment of $64 \times 23 \mathrm{~mm}^{2}$ is visible in the target element. On the right side, $\mathrm{CuCrZr}$ plates are visible on which the graphite baffle tiles will be mounted 


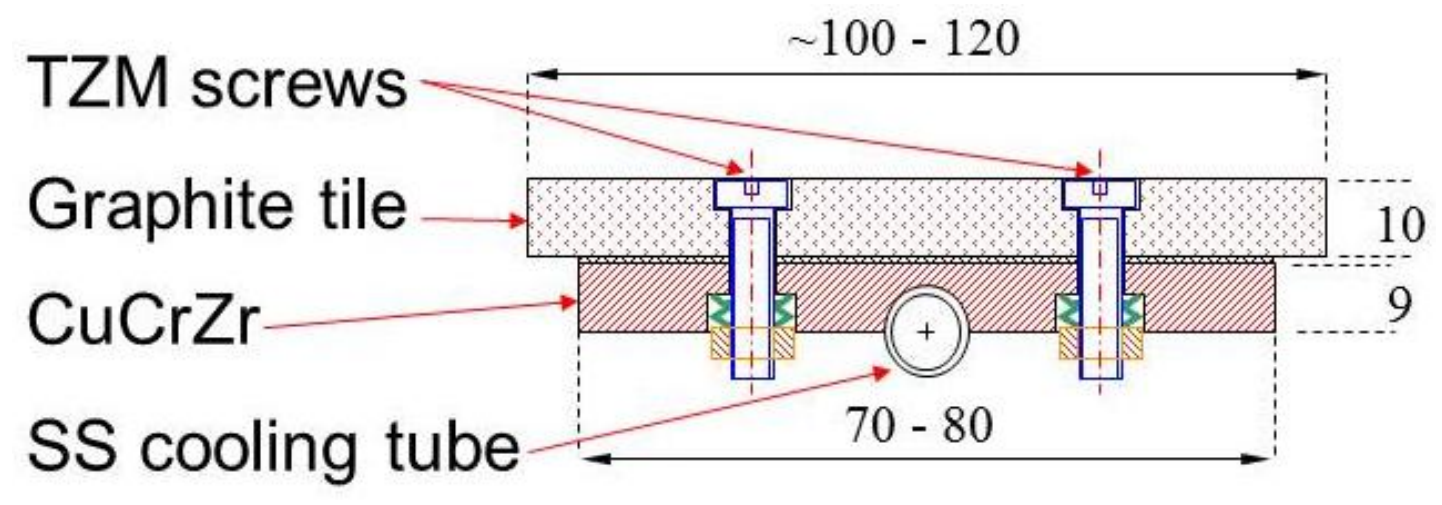

Figure 5. Schematics of a heat shield with graphite tile fixed on $\mathrm{CuCrZr}$ plate with a thin flexible graphite interlayer, using TZM screws. CuCrZr is brazed on SS cooling tube

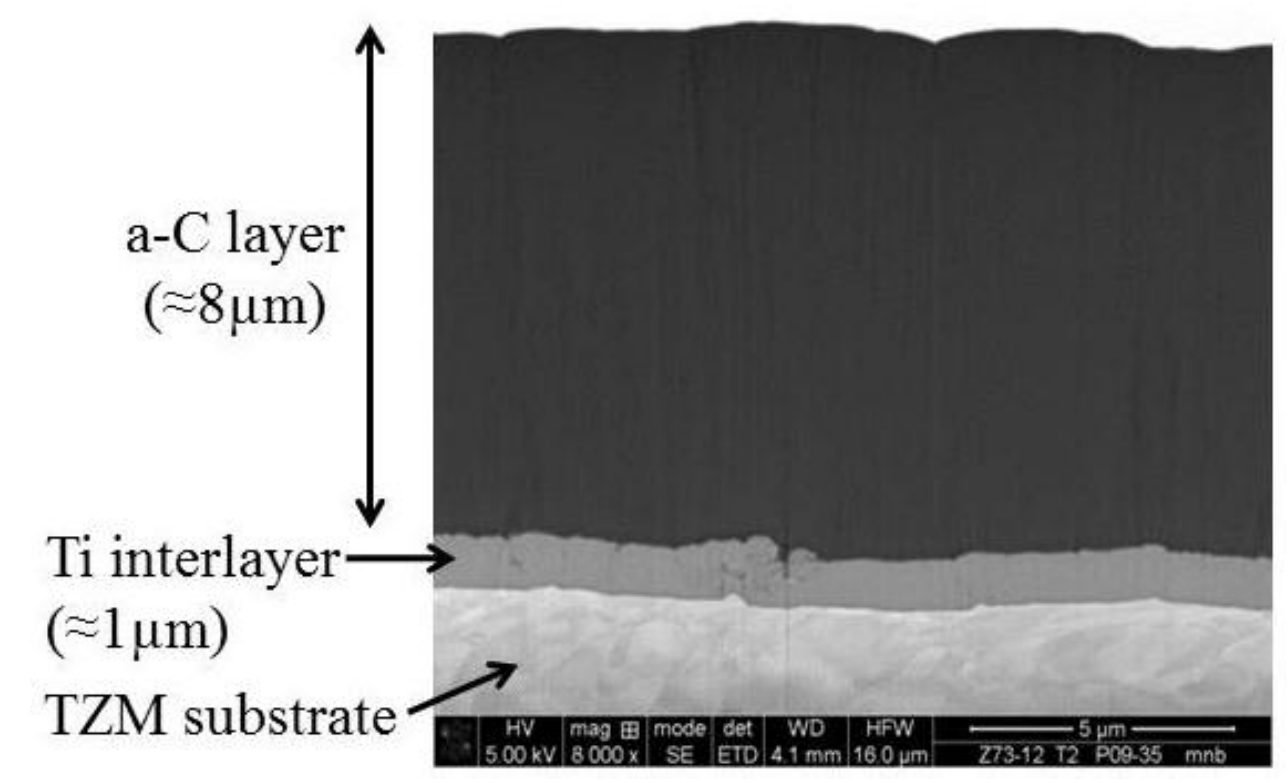

Figure 6. SEM image of the a-C coating on TZM with Ti interlayer 


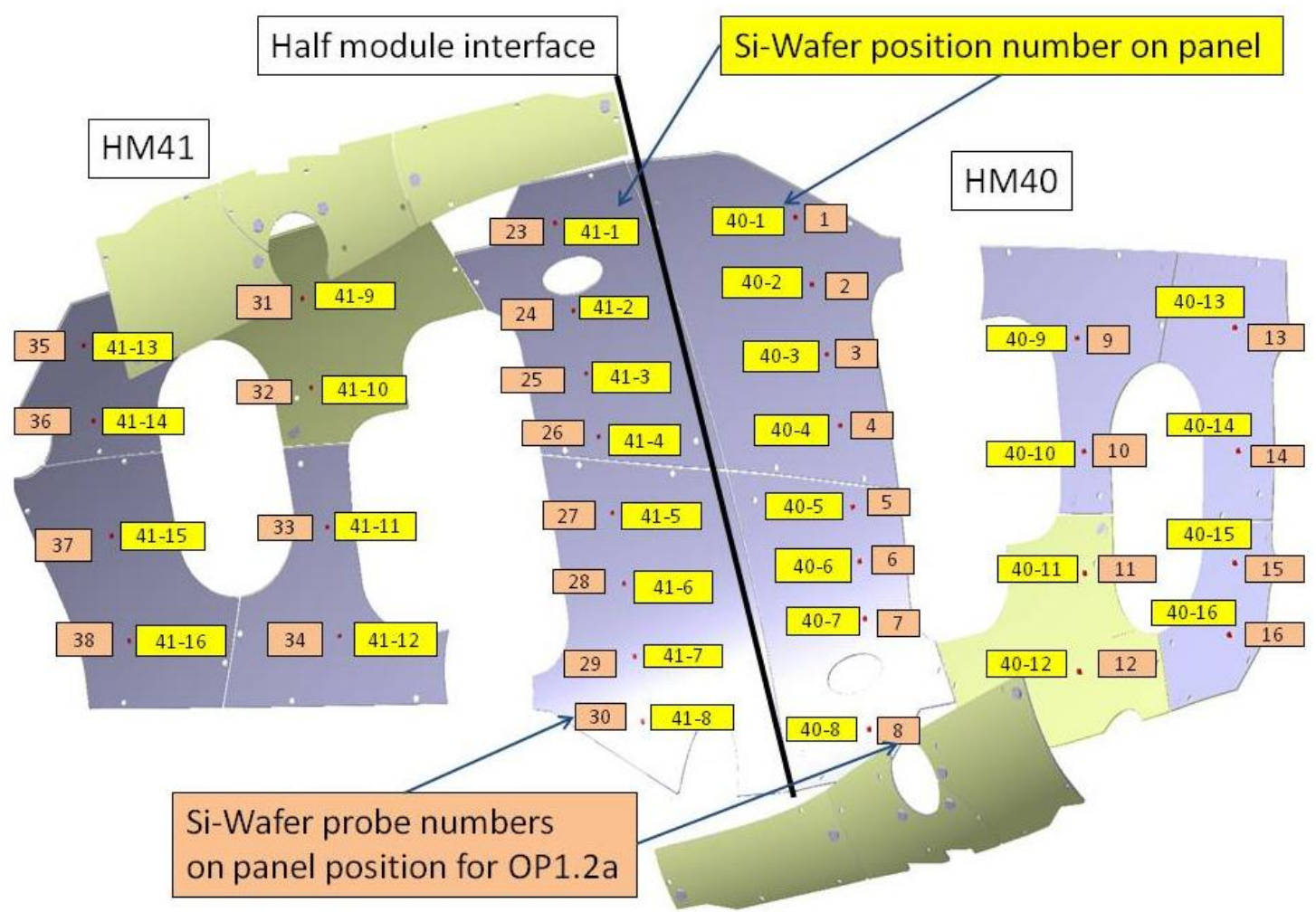

Figure 7. The mounting positions of $44 \mathrm{Si}$-wafer probes on the stainless steel wall panels of W7-X half modules 40 and 41 . At each location, the panel position number and the probe numbers are shown

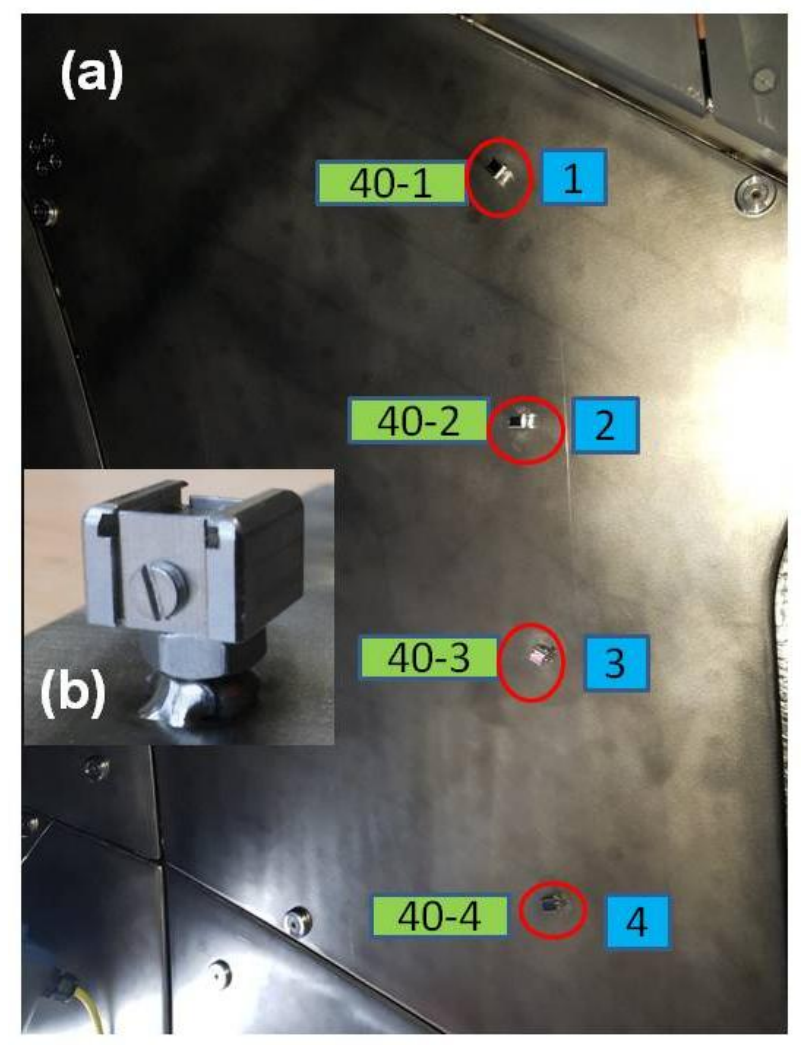

Figure 8. (a) View of the 4 pieces of Si wafer probes mounted on the outer SS wall panel, (b) view of the probe head $\left(11.5 \times 11.5 \times 8 \mathrm{~mm}^{3}\right)$ screwed on the bolt welded on panel 